\title{
Dynamic Response to Moving Distributed Masses of Pre- Stressed Uniform Rayleigh Beam Resting on Variable Elastic Pasternak Foundation
}

\section{Adeoye $\mathrm{AS}^{*}$ and Awodola TO}

\begin{abstract}
Affiliation: Department of Mathematical Sciences, Federal University of Technology, Akure, Nigeria
*Corresponding author: Adeoye Samuel Adebola, Department of Mathematical Sciences, Federal University of Technology, Akure, Nigeria, E-Mail: samueladebola84@gmail.com

Citation: Adeoye AS and Awodola TO. Dynamic response to moving distributed masses of pre-stressed uniform rayleigh beam resting on variable elastic pasternak foundation (2018) Edelweiss Appli Sci Tech 2: 1-9
\end{abstract}

Received: Nov 11, 2017

Accepted: Dec 18, 2017

Published: Jan 01, 2018

Copyright: @ 2018 Adeoye AS, et al., This is an open-access article distributed under the terms of the Creative Commons Attribution License, which permits unrestricted use, distribution, and reproduction in any medium, provided the original author and source are cre dited.

\begin{abstract}
The dynamic response to moving distributed masses of pre-stressed uniform Rayleigh beam resting on variable elastic Pasternak foundation is examined. The equation governing this problem is a fourth order partial differential equation with variable and singular co-efficients. To solve this cumbersome equation, the method of Galerkin approach is adopted to reduce the governing differential equation to a sequence of coupled second order ordinary differential equation which is then simplified further with modified asymptotic method of Struble. The more simplified equation is solved using the Laplace transformation technique. The closed form solutions obtained are analyzed in order to show the conditions of resonance, and to show that resonance is attained earlier in moving mass system than in the moving force system. The results in plotted graphs show that as the axial force, the rotatory inertia, foundation modulus and shear modulus increase, the deflection of the elastically supported nonuniform Rayleigh beam decreases in each case. The transverse deflections of the beam on variable Pasternak elastic foundation are higher under the action of moving masses than those when only the force effects of the moving load are considered. This implies that resonance is reached faster in moving mass problem than in moving force problem.
\end{abstract}

Keywords: Pasternak foundation, Shear deformation, Resonance, Critical speed, Natural frequency, Axial force, Modified frequency.

\section{Introduction}

Transport structures such as railway or bridges are subjected to moving vehicles (loads) which vary in both space and time. The branch of transport has experienced great advances, characterized by increasing high speed and weights of vehicles. These structures on which the vehicles move have been subjected to vibration and dynamic stress more than ever before. Therefore, the moving load problem has been a fundamental problem in several fields of applied mathematics, mechanical engineering, applied physics and railway engineering. Rails and bridges are examples of structured elements to be designed to support moving masses. Most importantly, problems of this type are mathematically cumbersome when the inertial effect of the load is taken into consideration. The challenges of these designs have attracted the interest of many researchers in the fields of applied mathematics, mechanical engineering, applied physics and railway engineering. Some of these researchers include Fryba [1], who studied the vibration of solids and structures under moving loads. Gbadeyan and Dada [2] examined the influence of elastic foundation on plate under a moving load without considering the influence of rotatory inertia and shear deformation on the plate. The work of Stanistic et al was taken up much later by Gbadeyan and Oni [3], who investigated the dynamic analysis of an elastic plate continuously supported by an elastic foundation and traversed by an arbitrary number of concentrated moving masses. Yavari [4], studied the generalized solution of beams with jump discontinuities on elastic foundation. Yin [5] also investigated the closed form solution for reinforced Timoshenko beam on elastic foundation. In the same vein, Teodoru [6] in his work, analyzed beam on elastic foundation by using finite difference approach.

Oni and Awodola [7] investigated the vibrations under a moving load of a non-uniform Rayleigh beam on variable elastic foundation. Oni and Awodola [8] also analyzed the dynamic response under a moving load of an elastically supported non-prismatic Bernoulli-Euler beam on variable elastic foundation. In the work of Oni and Omolofe [9], the dynamic analysis of a pre-stressed elastic beam with general boundary conditions under moving loads at varying velocities was investigated. The study on exact series solution for the transverse vibrations of rectangular plates with elastic boundary supports was carried out by Li [10]. Hsu [11], studied the vibration analysis of non-uniform beams resting on elastic foundation. The work of Ismail [12] on dynamic response of a beam due to an accelerating moving mass using moving finite element approximation cannot be ignored. Kargarmovin and Younesian [13] took

Citation: Adeoye AS and Awodola TO. Dynamic response to moving distributed masses of prestressed uniform rayleigh beam resting on variable elastic pasternak foundation (2018) Edelweiss Appli Sci Tech 2: 1-9 
further study on dynamic of Timoshenko beams on Pasternak foundation under moving load.

Recently, Adeoye and Awodola [14] took a close studied on influence of rotatory inertial correction factor on the vibration of elastically supported non-uniform Rayleigh beam using Galerkin method and Struble technique. Adeoye and Akintomide [15] investigated dynamic behavior of Bernoulli-Euler beam with elastically supported boundary conditions under moving distributed masses on constant bi-parametric foundation using Galerkin method and Struble technique. Akintomide and Awodola [16] analyzed the dynamic response to variable-magnitude moving distributed masses of Bernoulli-Euler beam on bi-parametric foundation and they obtained the closed form solution using Runge-Kutta technique.

In our recent research work, Adeoye and Awodola [14] effort was made to investigate the influence of rotatory inertial correction factor on the vibration of elastically supported non-uniform Rayleigh beam on variable foundation. The objective of this paper is to extend this research work to elastically supported uniform Rayleigh beam on variable elastic biparametric foundation. This paper therefore investigates dynamic response to moving distributed masses of pre-stressed uniform Rayleigh beam resting on variable elastic Pasternak foundation.

\section{Governing Equation}

Considering the dynamic response to moving distributed masses of prestressed uniform Rayleigh beam on variable elastic Pasternak foundation; the governing equation of motion is given by the fourth order partial differential equation Fryba [1].

$\mathcal{A} I \frac{\partial^{4}}{\partial x^{4}} b(x, t) \frac{\partial}{\partial x}\left[\mathcal{N}_{o} \frac{\partial}{\partial x} b(x, t)\right] \mathfrak{B}(x) \frac{\partial^{2}}{\partial t^{2}} b(x, t) \mathfrak{B}(x) \mathcal{R}_{o} \frac{\partial^{4}}{\partial x^{2} \partial t^{2}} b(x, t)$ $+\delta(x, t)=\mathcal{P}(x, t)(1)$

Where $x$ is the spatial co-ordinate, $\mathrm{t}$ is the time co-ordinate, $b(x, t)$ is the transverse displacement, $\mathcal{A} I$ is the flexural rigidity of the structure, $\mathfrak{B}(x)$ is the variable mass per unit length of the non-uniform beam, $\mathcal{N}_{\mathrm{o}}$ is the constant axial force, $\mathcal{R}_{o}$ is the rotatory inertial correction factor, $\delta(x, t)$ is the variable foundation reaction $\mathcal{P}(x, t)$ is the moving distributed load.

The relationship between the foundation reaction and lateral deflection $b(x, t)$ is

$\delta(x, t)=\mathcal{S}(x) b(x, t)-\frac{\partial}{\partial x}\left[\mathcal{H}(x) \frac{\partial}{\partial x} b(x, t)\right]$

Where, $\mathcal{S}(x)$ and $\mathcal{H}(x)$ are two variable parameters of the elastic foundation. That is, $\mathcal{S}(x)$ is the variable foundation stiffness (foundation modulus) and $\mathcal{H}(x)$ is the variable shear modulus.

Where

$$
\begin{array}{r}
\mathcal{S}(x)=\mathcal{S}_{O}\left(4 x-3 x^{2}+x^{3}\right) \\
\mathcal{H}(x)=\mathcal{H}_{O}\left(12-13 x+6 x^{2}+x^{3}\right) \\
\mathfrak{B}(x)=\mathfrak{B}_{o}\left(1+\sin \frac{\pi x}{L}\right)
\end{array}
$$

Substituting equations (3), (4) and (5) into equation (1), one obtains

$$
\begin{aligned}
& \mathrm{A} I \frac{\partial^{4}}{\partial x^{4}} b(x, t)-\mathrm{N} \frac{\partial^{2}}{\partial x^{2}} b(x, t)+\mathrm{B}_{o}\left(1+\sin \frac{\pi x}{L}\right) \frac{\partial^{2}}{\partial t^{2}} b(x, t) \\
& -\mathrm{B}_{o}\left(1+\sin \frac{\pi x}{L}\right) \mathrm{R}_{o} \frac{\partial^{4}}{\partial x^{2} \partial t^{2}} b(x, t)+\mathrm{S}_{o}\left(4 x-3 x^{2}+x^{3}\right) b(x, t) \\
& -\frac{\partial}{\partial x}\left[\mathrm{H}_{o}\left(12-13 x+6 x^{2}+x^{3}\right) \frac{\partial}{\partial x} b(x, t)\right]=\sum_{i=1}^{N} M g H(x-v t)\left[1-\frac{1}{g} \frac{d^{2}}{d t^{2}} b(x, t)\right]
\end{aligned}
$$

The boundary condition of the structure under consideration is first taken to be arbitrary and the initial condition without any loss of generality is taken as

$$
b(x, 0)=0=\frac{\partial}{\partial t} b(x, o)
$$

\section{Analytical Approximate Solution}

Due to complex nature of equation (1), no conventional method can be used to solve the partial differential equation and till this moment, there is no exact closed form solution to equation(1). Therefore, an approximate solution is sought. The method of Galerkin is used to reduce equation (1) to second order coupled ordinary differential equations, and this takes the form

$$
b_{i}(x, t)=\sum_{i=1}^{N} w_{i}(t) u_{i}(x)
$$

where

$$
\begin{aligned}
& U_{i}(x)=\sin \frac{\mathfrak{D}_{i} x}{L}+A_{i} \cos \frac{\mathfrak{D}_{i} x}{L}+B_{i} \sinh \frac{\mathfrak{D}_{i} x}{L}+C_{i} \cosh \frac{\mathfrak{D}_{i} x}{L} \\
& \sum_{m=1}^{N}\left[\left(\mathbf{U}_{i}(x)+\sin \frac{\pi x}{L} \mathbf{U}_{i}(x)-\mathbf{R}_{o}\left(\mathbf{U}_{i}^{\prime \prime}(x)+\sin \frac{\pi x}{L} \mathbf{U}_{i}^{\prime \prime}(x)\right)\right) W_{i}(t)+\right. \\
& \left.\left(\frac{\mathrm{A} I}{4 \mathrm{~B}_{o}} \mathrm{U}_{i}^{i v}(x)-\frac{\mathrm{N}_{o}}{\mathrm{~B}_{o}} \mathrm{U}_{i}^{\prime \prime}(x)+\frac{\mathrm{S}_{o}}{\mu_{0}}\left(4 x \mathrm{U}_{i}(x)-3 x^{2} \mathrm{U}_{i}(x)+x^{3} \mathrm{U}_{i}(x)\right)\right)\right) \\
& -\frac{\mathrm{H}_{o}}{\mathrm{~B}_{o}}\left(\left(\begin{array}{l}
-13 \mathrm{U}_{i}^{\prime}(x)+12 x \mathrm{U}_{i}^{\prime}(x)-3 x^{2} \mathrm{U}_{i}^{\prime}(x) \\
+12 \mathrm{U}_{i}^{\prime \prime}(x)-13 x \mathrm{U}_{i}^{\prime \prime}(x)+6 x^{2} \mathrm{U}_{i}^{\prime \prime}(x)-x^{3} \mathrm{U}_{i}^{\prime \prime}(x)
\end{array}\right)\right. \\
& w_{i}(t)+\frac{M}{\mathrm{~B}} H(x-v t)\left(\mathrm{U}_{i}(x) W_{i}(t)+2 v \mathrm{U}_{i}^{\prime}(x) w_{i}(t)+v^{2} \mathrm{U}_{i}^{\prime \prime}(x) w_{i}(t)\right) \\
& \left.-\frac{M g}{\mathrm{~B}_{o}} H(x-v t)\right]=0 \\
& \int_{0}^{L} \sum_{i=1}^{N}\left[\left(u_{i}(x) u_{j}(x)+\sin \frac{\pi x}{L} u_{i}(x) u_{j k}(x)\right.\right. \\
& \left.-\mathcal{R}_{o}\left(u_{i}^{\prime \prime}(x) u_{j}(x)+\sin \frac{\pi x}{L} u_{i}^{\prime \prime}(x) u_{j}(x)\right)\right) \ddot{w}_{i}(t) \\
& +\left(\frac{\mathcal{A} I}{4 \mathfrak{B}_{o}} u_{i}^{i v}(x) u_{j}(x)-\frac{\mathcal{N}_{o}}{\mathfrak{B}_{o}} u_{i}^{\prime \prime}(x) u_{j}(x)\right. \\
& +\frac{s_{0}}{\mathfrak{B}_{o}}\left(4 x u_{i}(x) u_{j}(x)-3 x^{2} u_{i}(x) u_{j}(x)+x^{3} u_{i}(x) u_{j}(x)\right) \\
& -\frac{\mathcal{H}_{0}}{\mathfrak{B}_{0}}\left[-13 u_{i}^{\prime}(x) u_{j}(x)+12 x u_{i}^{\prime}(x) u_{j}(x)-3 x^{2} u_{i}^{\prime}(x) u_{j}(x)\right. \\
& +12 u_{i}^{\prime \prime}(x) u_{j}(x)-13 x u_{i}^{\prime \prime}(x) u_{j}(x)+6 x^{2} u_{i}^{\prime \prime}(x) u_{j}(x) \\
& \left.\left.-x^{3} u_{i}^{\prime \prime}(x) U_{j}(x)\right]\right) w_{i}(t)+\frac{M}{\mathfrak{B}_{o}} H(x \\
& -v t)\left(u_{i}(x) u_{j}(x) \ddot{w}_{i}(t)+2 v u_{i}^{\prime}(x) u_{j}(x) \dot{w}_{i}(t)+v^{2} u_{i}^{\prime \prime}(x) u_{j}(x) w_{i}(t)\right) \\
& \left.-\frac{M}{\mathfrak{B}_{o}} g H(x-v t) u_{k}(x)\right] d x=0
\end{aligned}
$$


Equation (11) can be re-written as

$$
\begin{aligned}
& \sum_{i=1}^{N}\left[\left(E_{1}(i, j)+E_{2}(i, j)-\mathcal{R}_{o}\left(E_{3}(i, j)+E_{4}(i, j)\right)\right) \ddot{w}_{i}(t)\right. \\
+ & {\left[F_{A} E_{5}(i, j)-F_{B} E_{6}(i, j)+\frac{\delta_{O}}{\mathfrak{B}_{o}}\left(4 E_{7}(i, j)-3 E_{8}(i, j)+E_{9}(i, j)\right)\right.} \\
- & \frac{\mathcal{H}_{O}}{\mathfrak{B}_{o}}\left(-13 E_{10}(i, j)+12 E_{11}(i, j)-3 E_{12}(i, j)+12 E_{13}(i, j)-13 E_{14}(i, j)\right. \\
+ & \left.\left.6 E_{15}(i, j)-3 E_{16}(i, j)\right)\right] w_{i}(t) \\
+ & \left.\frac{M}{\mu_{0}}\left[E_{17}(i, j) \ddot{w}_{i}(t)+2 v E_{18}(i, j) \dot{w}_{i}(t)+v^{2} E_{19}(i, j) w_{i}(t)\right]-\frac{M g}{\mu_{0}} B_{20}(j)\right] \\
= & 0
\end{aligned}
$$

Where

$$
\begin{aligned}
& F_{A}=\frac{\mathcal{A} I}{4 \mathfrak{B}_{o}}, E_{1}(i, j)=\int_{0}^{L} U_{i}(x) U_{j}(x) d x, E_{2}(i, j)=\int_{0}^{L} \sin \frac{\pi x}{L} U_{i}(x) U_{j}(x) d x \\
& E_{3}(i, j)=\int_{0}^{L} u_{i}^{\prime \prime}(x) u_{j}(x) d x, E_{4}(i, j)=\int_{0}^{2} \sin \frac{\pi x}{L} u_{i}^{\prime \prime}(x) u_{j}(x) d x \\
& E_{5}(i, j)=\int_{0}^{2} u_{i}^{i v}(x) u_{j}(x) d x, \quad E_{6}(i, j)=\int_{0}^{2} u_{i}^{\prime \prime}(x) u_{j}(x) d x \\
& E_{7}(i, j)=\int_{0}^{2} x u_{i}(x) u_{j}(x) d x, \quad E_{8}(i, j)=\int_{0}^{2} x^{2} u_{i}(x) u_{j}(x) d x \\
& E_{9}(i, j)=\int_{0}^{L} x^{3} u_{i}(x) u_{j}(x) d x, \quad E_{10}(i, j)=\int_{0}^{L} u_{i}^{\prime}(x) u_{j}(x) d x \\
& E_{11}(i, j)=\int_{0}^{2} x u_{i}^{\prime}(x) u_{j}(x) d x, \quad E_{12}(i, j)=\int_{0}^{2} x^{2} u_{i}^{\prime}(x) u_{j}(x) d x \\
& E_{13}(i, j)=\int_{0}^{2} u_{i}^{\prime \prime}(x) u_{j}(x) d x, \quad E_{14}(i, j)=\int_{0}^{2} x u_{i}^{\prime \prime}(x) u_{j}(x) d x \\
& E_{15}(i, j)=\int_{0}^{2} x^{2} u_{i}^{\prime \prime}(x) u_{j}(x) d x, \quad E_{16}(i, j)=\int_{0}^{L} x^{3} u_{i}^{\prime \prime}(x) u_{j}(x) d x \\
& E_{17}(i, j)=\int_{0}^{L} H(x-v t) u_{i}(x) u_{j}(x) d x, \quad E_{18}(i, j)=\int_{0}^{L} H(x-v t) u_{i}^{\prime}(x) u_{j}(x) d x \quad \text { (21) } \\
& E_{19}(i, j)=\int_{0}^{2} H(x-v t) U_{i}^{\prime \prime}(x) U_{j}(x) d x, E_{20}(j)=\int_{0}^{2} H(x-v t) U_{j}(x) d x, F_{B}=\frac{\mathcal{N}_{0}}{\mathfrak{B}_{0}}(22)
\end{aligned}
$$

In order to evaluate the integrals in E_17 (i,j),E_18 (i,j) and E_19 (i,j), one makes use of the Fourier series representation for the Heaviside function in the form;

$$
H(x-v t)=\frac{1}{4}+\frac{1}{\pi} \sum_{n=1}^{\infty} \frac{\sin (2 n+1) \pi(x-v t)}{2 n+1}, 0<x<1
$$

To solve $E_{20}(i, j)$, one makes use of the definition of Heaviside function and substitute the result into (12), one obtains,

$$
\begin{gathered}
\sum_{i=1}^{N}\left[\left(E_{1}(i, j)+E_{2}(i, j)-\mathcal{R}_{o}\left(E_{3}(i, j)+E_{4}(i, j)\right)\right) \ddot{w}_{i}(t)+\left[F_{A} E_{5}(i, j)-F_{B} E_{6}(i, j)+\right.\right. \\
\frac{\delta_{o}}{\mathfrak{B}_{o}}\left(4 E_{7}(i, j)-3 E_{8}(i, j)+E_{9}(i, j)\right)-\frac{\mathcal{H}_{o}}{\mathfrak{B}_{o}}\left(-13 E_{10}(i, j)+12 E_{11}(i, j)-3 E_{12}(i, j)+\right. \\
\left.\left.12 E_{13}(i, j)-13 E_{14}(i, j)+6 E_{15}(i, j)-3 E_{16}(i, j)\right)\right] \omega_{i}(t)+\frac{M}{\mathfrak{B}_{o}}\left[E_{17}(i, j) \ddot{w}_{i}(t)+\right. \\
\left.\left.2 v E_{18}(i, j) \dot{w}_{i}(t)+v^{2} E_{19}(i, j) w_{i}(t)\right]\right]=\frac{M g L}{\mathfrak{B}_{o} \mathfrak{D}_{j}}\left[-\cos \mathfrak{D}_{j}+A_{j} \sin \mathfrak{D}_{j}+B_{j} \cosh \mathfrak{D}_{j}+C_{j} \sinh \mathfrak{D}_{j}+\right. \\
\left.\cos \frac{\mathfrak{D}_{j} v t}{L}-A_{j} \sin \frac{\mathfrak{D}_{j} v t}{L}-B_{j} \cosh \frac{\mathfrak{D}_{j} v t}{L}-C_{j} \sinh \frac{\mathfrak{D}_{j} v t}{L}\right]
\end{gathered}
$$

Therefore, equation (24) becomes

$$
\begin{aligned}
& 5_{0}(i, j) \ddot{w}_{i}(t)+5_{1}(i, j) w_{i}(t)+\varpi\left[\left(\frac{1}{4} G_{1}(i, j)\right.\right. \\
& \left.+\frac{1}{\pi} \sum_{n=0}^{\infty} \cos \frac{(2 n+1) \pi v t}{2 n+1} \mathcal{G}_{2}(n, i, j)-\frac{1}{\pi} \sum_{n=0}^{\infty} \sin \frac{(2 n+1) \pi v t}{2 n+1} \mathcal{G}_{3}(n, i, j)\right) \ddot{w}_{i}(t) \\
& +2 c\left(\frac{1}{4} \mathcal{G}_{4}(i, j)+\frac{1}{\pi} \sum_{n=0}^{\infty} \cos \frac{(2 n+1) \pi v t}{2 n+1} \mathcal{G}_{5}(n, i, j)\right. \\
& \left.-\frac{1}{\pi} \sum_{n=0}^{\infty} \sin \frac{(2 n+1) \pi v t}{2 n+1} \mathcal{G}_{6}(n, i, j)\right) \dot{w}_{i}(t)+c^{2}\left(\frac{1}{4} \mathcal{G}_{7}(i, j)\right. \\
& \left.+\frac{1}{\pi} \sum_{n=0}^{\infty} \cos \frac{(2 n+1) \pi v t}{2 n+1} \mathcal{G}_{8}(n, i, j)-\frac{1}{\pi} \sum_{n=0}^{\infty} \sin \frac{(2 n+1) \pi v t}{2 n+1} \mathcal{G}_{9}(n, i, j)\right) w_{i}(t) \\
& =\frac{P L}{\mathfrak{B}_{o} \mathfrak{D}_{j}}\left[-\cos \mathfrak{D}_{j}+A_{j} \sin \mathfrak{D}_{j}+B_{j} \cosh \mathfrak{D}_{j}+C_{j} \sinh \mathfrak{D}_{j}+\cos \frac{\mathfrak{D}_{j} v t}{L}-A_{j} \sin \frac{\mathfrak{D}_{j} v t}{L}-B_{j} \cosh \frac{\mathfrak{D}_{j} v t}{L}-\right. \\
& \left.C_{k} \sinh \frac{D_{j} v t}{L}\right]
\end{aligned}
$$

Where

$$
\begin{gathered}
\overline{5}_{0}(i, j)=\left[E_{1}(i, j)+E_{2}(i, j)-\mathcal{R}_{o}\left(E_{3}(i, j)+E_{4}(i, j)\right)\right] \\
5_{1}(i, j)=F_{A} E_{5}(i, j)-F_{B} E_{6}(i, j)+\frac{\mathcal{S}_{o}}{\mathfrak{B}_{o}}\left(4 E_{7}(i, j)-3 E_{8}(i, j)+E_{9}(i, j)\right) \\
\quad-\frac{\mathcal{H}_{o}}{\mathfrak{B}_{o}}\left(-13 E_{10}(i, j)+12 E_{11}(i, j)-3 E_{12}(i, j)+12 E_{13}(i, j)-13 E_{14}(i, j)\right. \\
\left.\quad+6 E_{15}(i, j)-3 E_{16}(i, j)\right) \\
\begin{array}{ll}
\varpi=\frac{\mathrm{M}}{\mathfrak{B}_{o} L}, \quad P & M g
\end{array}
\end{gathered}
$$

Equation (25) is re-written as

$$
\begin{aligned}
& \ddot{w}_{i}(t)+\frac{5_{1}(i, j)}{5_{0}(i, j)} w_{i}(t)+ \\
& \varpi\left[\left(\frac{1}{4} \frac{G_{1}(i, j)}{5_{0}(i, j)}+\frac{1}{\pi} \sum_{n=0}^{\infty} \cos \frac{(2 n+1) \pi v t}{2 n+1} \frac{G_{2}(i, j)}{5_{0}(i, j)}-\frac{1}{\pi} \sum_{n=0}^{\infty} \sin \frac{(2 n+1) \pi v t}{2 n+1} \frac{G_{3}(i, j)}{5_{0}(i, j)}\right) \ddot{w}_{i}(t)+2 v\left(\frac{1}{4} \frac{G_{4}(i, j)}{5_{0}(i, j)}+\right.\right. \\
& \left.\frac{1}{\pi} \sum_{n=0}^{\infty} \cos \frac{(2 n+1) \pi v t}{2 n+1} \frac{G_{5}(i, j)}{5_{0}(i, j)}-\frac{1}{\pi} \sum_{n=0}^{\infty} \sin \frac{(2 n+1) \pi v t}{2 n+1} \frac{G_{6}(i, j)}{5_{0}(i, j)}\right) w_{i}(t)+ \\
& \left.v^{2}\left(\frac{1}{4} \frac{G_{7}(i, j)}{5_{0}(i, j)}+\frac{1}{\pi} \sum_{n=0}^{\infty} \cos \frac{(2 n+1) \pi v t}{2 n+1} \frac{\mathcal{G}_{8}(i, j)}{5_{0}(i, j)}-\frac{1}{\pi} \sum_{n=0}^{\infty} \sin \frac{(2 n+1) \pi v t}{2 n+1} \frac{\mathcal{G}_{9}(i, j)}{5_{0}(i, j)}\right) w_{i}(t)\right] \\
& =\frac{P L}{\mathfrak{B}_{0} \mathfrak{D}_{j} \mathfrak{D}_{0}(i, j)}\left[-\cos \mathfrak{D}_{j}+A_{j} \sin \mathfrak{D}_{j}+B_{j} \cosh \mathfrak{D}_{j}+C_{j} \sinh \mathfrak{D}_{j}+\cos \frac{\mathfrak{D}_{j} v t}{L}-A_{j} \sin \frac{\mathfrak{D}_{j} v t}{L}-\right. \\
& \left.B_{j} \cosh \frac{\mathfrak{D}_{j} v t}{L}-C_{j} \sinh \frac{\mathfrak{D}_{j} v t}{L}\right] \\
& \text { (29) }
\end{aligned}
$$

Equation (29) is the transformed equation governing the problem of supported beam on variable bi-parametric elastic foundation. This coupled non-homogeneous second order ordinary differential equation is assumed to have arbitrary boundary conditions.

\section{Case I: Moving Force Problem}

In moving force problem, only the load is being transferred to the structure. In this case, the inertia effect is negligible. Setting $\varpi=0$ in the transformed equation (27), one obtains

$$
\begin{gathered}
\ddot{w}_{i}(t)+\frac{5_{1}(i, j)}{5_{o}(i, j)} w_{i}(t)=\frac{P L}{\mathfrak{B}_{o} \mathfrak{D}_{j} 5_{o}(i, j)}\left[-\cos \mathfrak{D}_{j}+A_{j} \sin \mathfrak{D}_{j}+B_{j} \cosh \mathfrak{D}_{j}+C_{j} \sinh \mathfrak{D}_{j}\right. \\
\left.+\cos \frac{\mathfrak{D}_{j} v t}{L}-A_{j} \sin \frac{\mathfrak{D}_{j} v t}{L}-B_{j} \cosh \frac{\mathfrak{D}_{j} v t}{L}-C_{j} \sinh \frac{\mathfrak{D}_{j} v t}{L}\right]
\end{gathered}
$$

Equation (28) can be rewritten as,

$$
\begin{aligned}
& \ddot{w}_{i}(t)+\sigma_{i}^{2} w_{i}(t)=\frac{P L}{\mathfrak{B}_{o} \mathfrak{D}_{j} \mathfrak{L}_{o}(i, j)}\left[\psi_{j}+\cos \frac{\mathcal{D}_{j} v t}{L}-A_{j} \sin \frac{\mathfrak{D}_{j} v t}{L}-B_{j} \cosh \frac{\mathfrak{D}_{j} v t}{L}\right. \\
& \left.-C_{j} \sinh \frac{\mathcal{D}_{j} v t}{L}\right]
\end{aligned}
$$

Citation: Adeoye AS and Awodola TO. Dynamic response to moving distributed masses of pre-stressed uniform rayleigh beam resting on variable elastic pasternak foundation (2018) Edelweiss Appli Sci Tech 2: $1-9$ 
Where

$$
\sigma_{i}^{2}=\frac{\overline{5}_{1}(i, j)}{\mathfrak{5}_{0}(i, j)} \text { and } \psi_{j}=-\cos \mathfrak{D}_{j}+A_{j} \sin \mathfrak{D}_{j}+B_{j} \cosh \mathfrak{D}_{j}+C_{j} \sinh \mathfrak{D}_{j}
$$

Equation (29) is an approximate model, which assumes the inertia effect of the moving mass as negligible.

Further rearrangement of (29) yields

$$
\dddot{w}_{i}(t)+\sigma_{i}^{2} w_{i}(t)=\frac{P L}{\mathfrak{B}_{o} \mathfrak{D}_{k} 5_{0}(i, j)}\left[\psi_{j}+\cos \Omega_{j} t-A_{j} \sin \Omega_{j} t-B_{j} \cosh \Omega_{j} t-C_{j} \sinh \Omega_{j} t\right]
$$

Where

$$
\Omega_{j}=\frac{\mathfrak{D}_{j} v}{L}
$$

Solving equation (31) using Laplace transformation techniques and taking into account equation (7) one obtains

$$
\begin{aligned}
b_{i}(x, t)= & \frac{P L}{\mathfrak{B}_{o} \mathfrak{D}_{j} \mathfrak{S}_{0}(i, j)} \times \frac{1}{\sigma_{i}^{2}\left(\sigma_{i}^{4}-\Omega_{j}^{4}\right)}\left[\beta_{j}\left(1-\cos \sigma_{i} t\right)\left(\sigma_{i}^{4}-\Omega_{j}^{4}\right)+\sigma_{i}^{2}\left(\sigma_{i}^{2}+\Omega_{j}^{2}\right)\left(\cos \Omega_{j} t\right.\right. \\
& \left.-\cos \sigma_{i} t\right)-A_{j} \sigma_{i}\left(\sigma_{i}^{2}+\Omega_{j}^{2}\right)\left(\sigma_{i} \sin \Omega_{j} t-\Omega_{j} \sin \sigma_{i} t\right)-B_{j} \sigma_{i}^{2}\left(\sigma_{i}^{2}-\Omega_{j}^{2}\right)\left(\cosh \Omega_{j} t\right. \\
& \left.\left.-\cos \sigma_{i} t\right)-C_{j} \sigma_{i}\left(\sigma_{i}^{2}+\Omega_{j}^{2}\right)\left(\sigma_{i} \sinh \Omega_{j} t-\Omega_{j} \sin \sigma_{i} t\right)\right] \\
& \times\left(\sin \frac{\mathfrak{D}_{i} x}{L}+A_{i} \cos \frac{\mathfrak{D}_{i} x}{L}+B_{i} \sinh \frac{\mathfrak{D}_{i} x}{L}+C_{i} \cosh \frac{\mathfrak{D}_{i} x}{L}\right)
\end{aligned}
$$

Equation (33) represents the transverse deflection of uniform Rayleigh beam under moving distributed force and resting on variable Pasternak elastic foundation.

\section{Case II: Moving Mass Problem}

In moving mass problem, the moving load is assumed to be rigid, and the weight and as well as inertia forces are transferred to the moving load. That is the inertia effect is not negligible. Thus $\varpi \neq 0$ and so it is required to solve the entire equation (27). Thus, equation (27) takes the form

$$
\begin{aligned}
& \ddot{w}_{i}(t)+\frac{F_{1}(i j)}{\xi_{0}(i, j)} w_{i}(t)+ \\
& \varpi\left[\left(\frac{1}{4} \frac{G_{1}(i, j)}{5_{0}(i, j)}+\frac{1}{\pi} \sum_{n=0}^{\infty} \cos \frac{(2 n+1) \pi v t}{2 n+1} \frac{G_{2}(i, j)}{5_{0}(i, j)}-\frac{1}{\pi} \sum_{n=0}^{\infty} \sin \frac{(2 n+1) \pi v t}{2 n+1} \frac{G_{3}(i, j)}{5_{0}(i, j)}\right) \ddot{w}_{i}(t)+2 v\left(\frac{1}{4} \frac{G_{6}(i, j)}{4} \frac{1(i, j)}{5_{0}}+\right.\right. \\
& \left.\frac{1}{\pi} \sum_{n=0}^{\infty} \cos \frac{(2 n+1) \pi v t}{2 n+1} \frac{G_{5}(i, j)}{5_{0}(i, j)}-\frac{1}{\pi} \sum_{n=0}^{\infty} \sin \frac{(2 n+1) \pi v t}{2 n+1} \frac{G_{6}(i, j)}{5_{0}(i, j)}\right) w_{i}(t)+ \\
& \left.v^{2}\left(\frac{1}{4} \frac{G_{7}(i, j)}{\xi_{0}(i, j)}+\frac{1}{\pi} \sum_{n=0}^{\infty} \cos \frac{(2 n+1) \pi v t}{2 n+1} \frac{G_{8}(i, j)}{5_{0}(i, j)}-\frac{1}{\pi} \sum_{n=0}^{\infty} \sin \frac{(2 n+1) \pi v t}{2 n+1} \frac{G_{9}(i, j)}{5_{0}(i, j)}\right) w_{i}(t)\right] \\
& =\frac{P L}{\mathfrak{B}_{0} \mathfrak{D}_{j} \mathfrak{F}_{0}(i, j)}\left[-\cos \mathfrak{D}_{j}+A_{j} \sin \mathfrak{D}_{j}+B_{j} \cosh \mathfrak{D}_{j}+C_{j} \sinh \mathfrak{D}_{j}+\cos \frac{\mathfrak{D}_{j} v t}{L}-A_{j} \sin \frac{\mathfrak{D}_{j} v t}{L}-\right. \\
& \left.B_{j} \cosh \frac{D_{j} v t}{L}-C_{j} \sinh \frac{D_{j} v t}{L}\right]
\end{aligned}
$$

On further rearrangements, one obtains

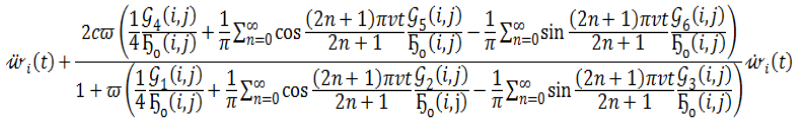

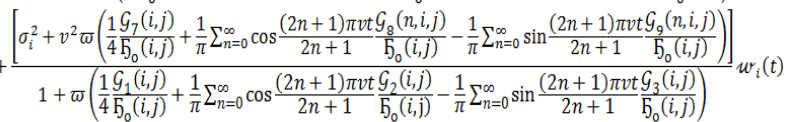

$$
\begin{aligned}
& P L \\
& =\frac{\overline{\mathfrak{B}_{0} \mathfrak{D}_{j} 5_{0}(i, j)}}{1+\omega\left(\frac{1}{4} \frac{\mathcal{G}_{1}(i, j)}{\overline{5}_{0}(i, j)}+\frac{1}{\pi} \sum_{n=0}^{\infty} \cos \frac{(2 n+1) \pi v t}{2 n+1} \frac{\mathcal{G}_{2}(i, j)}{\overline{5}_{0}(i, j)}-\frac{1}{\pi} \sum_{n=0}^{\infty} \sin \frac{(2 n+1) \pi v t}{2 n+1} \frac{\mathcal{G}_{3}(i, j)}{5_{0}(i, j)}\right)}\left[\psi_{j}\right. \\
& \left.+\cos \Omega_{j} t-A_{j} \sin \Omega_{j} t-B_{j} \cosh \Omega_{j} t-C_{j} \sinh \Omega_{j} t\right]
\end{aligned}
$$

Obviously, unlike the moving force problem, an exact analytical solution to equation (35) is not possible. In order to obtain approximate analytical solution, one makes use of a modification of the asymptotic method of Struble. By this method, one seeks the modified frequency corresponding to the frequency of the free system due to the presence of the effect of the moving mass. An equivalent system operator defined by the modified frequency then replaces equation (35).

We shall consider a parameter $\varpi_{0}<1$ for any arbitrary mass ratio defined by

$$
\varpi_{0}=\frac{\varpi}{1+\varpi}
$$

By using binomial theorem and truncating after second terms, one obtains $\quad \varpi_{0}=\varpi-o\left(\varpi^{2}\right)(37)$

Equation (45) becomes $\quad \varpi=\varpi_{0}$

to $o(\varpi)$ only and from equation (38)

$$
\frac{1}{1+\varpi_{o}\left(\frac{1}{4} \frac{\mathcal{G}_{1}(i, j)}{5_{\mathrm{o}}(i, j)}+\frac{1}{\pi} \sum_{n=0}^{\infty} \cos \frac{(2 n+1) \pi v t}{2 n+1} \frac{\mathcal{G}_{2}(i, j)}{5_{\mathrm{o}}(i, j)}-\frac{1}{\pi} \sum_{n=0}^{\infty} \sin \frac{(2 n+1) \pi v t}{2 n+1} \frac{\mathcal{G}_{3}(i, j)}{5_{\mathrm{o}}(i, j)}\right)}
$$

$$
\left[1-\varpi_{\mathrm{o}}\left(\frac{1}{4} \frac{\mathcal{G}_{1}(i, j)}{5_{\mathrm{o}}(i, j)}+\frac{1}{\pi} \sum_{n=0}^{\infty} \cos \frac{(2 n+1) \pi v t}{2 n+1} \frac{\mathcal{G}_{2}(i, j)}{5_{\mathrm{o}}(i, j)}-\right.\right.
$$

$\left.\left.\frac{1}{\pi} \frac{1}{\pi} \sum_{n=0}^{\infty} \sin \frac{(2 n+1) \pi v t}{2 n+1} \frac{\mathcal{G}_{3}(i, j)}{5_{0}(i, j)}\right)+\cdots\right]$

Where

\section{$<1 \quad(41)$}

Substituting equations (40) and (41) into equation (35), one obtains

$$
\begin{aligned}
& \ddot{w}_{i}(t)+2 c \varpi_{0}\left(\frac{1}{4} \frac{G_{4}(i, j)}{5_{0}(i, j)}+\frac{1}{\pi} \sum_{n=0}^{\infty} \cos \frac{(2 n+1) \pi v t}{2 n+1} \frac{G_{5}(i, j)}{\xi_{0}(i, j)}-\frac{1}{\pi} \sum_{n=0}^{\infty} \sin \frac{(2 n+1) \pi v t}{2 n+1} \frac{G_{6}(i, j)}{5_{0}(i, j)}\right) \dot{w}_{m}(t)+ \\
& {\left[\sigma_{i}^{2}-\right.} \\
& \sigma_{i}^{2} \varpi_{0}\left(\frac{1}{4} \frac{G_{1}(i, j)}{5_{0}(i, j)}+\frac{1}{\pi} \sum_{n=0}^{\infty} \cos \frac{(2 n+1) \pi v t}{2 n+1} \frac{G_{2}(i, j)}{5_{0}(i, j)}-\frac{1}{\pi} \sum_{n=0}^{\infty} \sin \frac{(2 n+1) \pi v t}{2 n+1} \frac{G_{3}(i, j)}{5_{0}(i, j)}\right)+c^{2} \varpi_{0}\left(\frac{1}{4} \frac{G_{7}(i, j)}{5_{0}(i, j)}+\right. \\
& \left.\left.\frac{1}{\pi} \sum_{n=0}^{\infty} \cos \frac{(2 n+1) \pi v t}{2 n+1} \frac{\mathcal{G}_{8}(i, j)}{5_{0}(i, j)}-\frac{1}{\pi} \sum_{n=0}^{\infty} \sin \frac{(2 n+1) \pi v t}{2 n+1} \frac{G_{g}(i, j)}{\zeta_{0}(i, j)}\right)\right] w_{m}(t)=\frac{P L}{\mathfrak{B}_{0} \mathbb{D}_{j} \mathrm{~F}_{0}(i, j)}\left[\psi_{j}+\cos \Omega_{j} t-\right. \\
& \left.\left.A_{j} \sin \Omega_{j} t-B_{j} \cosh \Omega_{j} t-C_{j} \sinh \Omega_{j} t\right]\right]
\end{aligned}
$$

Applying method of Struble technique to eqation (42) one obtains

$$
\begin{aligned}
& \frac{d^{2}}{d t^{2}} w_{i}(t)+\sigma_{i i}^{2} w_{i}(t)=\frac{P L}{\mathcal{B}_{o} \mathfrak{D}_{j} \mathfrak{L}_{o}(i, j)}\left[-\cos \mathcal{D}_{j}+A_{j} \sin \mathfrak{D}_{j}+B_{j} \cosh \mathcal{D}_{j}+C_{j} \sinh \mathcal{D}_{j}\right. \\
& \left.+\cos \frac{\mathfrak{D}_{j} v t}{L}-A_{j} \sin \frac{\mathcal{D}_{j} v t}{L}-B_{j} \cosh \frac{\mathfrak{D}_{j} v t}{L}-C_{j} \sinh \frac{\mathfrak{D}_{j} v t}{L}\right]
\end{aligned}
$$

Where

$$
\sigma_{i i}=\sigma_{i}-\frac{\varpi_{0}}{2}\left[\frac{\mathcal{G}_{1}(i, j) \sigma_{i}^{2}-v^{2} \mathcal{G}_{7}(i, j)}{4 \sigma_{i} 5_{0}(i, j)}\right]
$$

Citation: Adeoye AS and Awodola TO. Dynamic response to moving distributed masses of pre-stressed uniform rayleigh beam resting on variable elastic pasternak foundation (2018) Edelweiss Appli Sci Tech 2: 
Solving equation (43) using Laplace transformation techniques and taking into account equation (7) one obtains

$$
\begin{aligned}
b_{i}(x, t)= & \frac{P L}{\mathfrak{B}_{o} \mathfrak{D}_{j} \mathfrak{5}_{\mathrm{o}}(i, j)} \times \frac{1}{\sigma_{i i}^{2}\left(\sigma_{i i}^{4}-\Omega_{j}^{4}\right)}\left[\beta_{j}\left(1-\cos \sigma_{i i} t\right)\left(\sigma_{i i}^{4}-\Omega_{j}^{4}\right)+\sigma_{i i}^{2}\left(\sigma_{i i}^{2}+\Omega_{j}^{2}\right)\left(\cos \Omega_{j} t\right.\right. \\
& \left.-\cos \sigma_{i i} t\right)-A_{k} \sigma_{i i}\left(\sigma_{i i}^{2}+\Omega_{j}^{2}\right)\left(\sigma_{i i} \sin \Omega_{j} t-\Omega_{j} \sin \sigma_{i i} t\right)-B_{j} \sigma_{i i}^{2}\left(\sigma_{i i}^{2}\right. \\
& \left.\left.-\Omega_{j}^{2}\right)\left(\cosh \Omega_{j} t-\cos \sigma_{i i} t\right)-C_{j} \sigma_{i}\left(\sigma_{i i}^{2}+\Omega_{j}^{2}\right)\left(\sigma_{i i} \sinh \Omega_{j} t-\Omega_{j} \sin \sigma_{i i} t\right)\right] \\
& \times\left(\sin \frac{\mathfrak{D}_{i} x}{L}+A_{i} \cos \frac{\mathfrak{D}_{i} x}{L}+B_{i} \sinh \frac{\mathfrak{D}_{i} x}{L}+C_{i} \cosh \frac{\mathfrak{D}_{i} x}{L}\right)
\end{aligned}
$$

Equation (45) represents the transverse deflection of uniform Rayleigh beam under moving distributed mass and resting on variable Pasternak elastic foundation.

\section{Discussion of the Analytical Solutions}

For this undamped system, it is desirable to examine the phenomenon of

\begin{tabular}{|c|c|}
\hline & $\sigma_{i}=\Omega_{j}$ \\
\hline Where & $\Omega_{j}=\frac{\mathfrak{D}_{j} v}{L}$ \\
\hline that is & $\sigma_{i}=\frac{\mathfrak{D}_{j} v}{L}$ \\
\hline
\end{tabular}
resonance. From equation(33), it is clearly shown that the beam resting on variable bi-parametric elastic foundation and traversed by a moving distributed force reaches a state of resonance whenever

Equation (45) shows that the same beam under the action of moving distributed mass experiences resonance effect whenever

$$
\sigma_{i i}=\frac{\mathfrak{D}_{j} v}{L}
$$

From equation (44)

$$
\sigma_{i i}=\sigma_{i}-\frac{\varpi}{2}\left[\frac{\mathcal{G}_{1}(i, j) \sigma_{i}^{2}-v^{2} \mathcal{G}_{7}(i, j)}{4 \sigma_{i} 5_{0}(i, j)}\right]=\frac{\mathfrak{D}_{j} v}{L}
$$

It is therefore clear that for the same natural frequency, the critical speed for the system consisting of elastically supported uniform Rayleigh beam resting on variable elastic foundation and traverse by moving distributed force with uniform speed is greater than that of moving distributed mass problem. Thus for the same natural frequency, resonance is reached faster in the moving distributed mass system than in the moving distributed force system.

\section{Illustrative Examples}

\section{Clamped-Elastic Boundary Conditions}

At a clamped end, both deflection and slope vanish. Thus, when the Rayleigh beam is clamped at $x=0$ and elastically supported at $x=L$, the conditions are expressed as

$$
b(0, t)=0=b^{\prime}(0, t)
$$

at the end $x=0$ and

$$
b^{\prime \prime}-k_{1} b^{\prime}(L, t)=0=b^{\prime \prime \prime}(L, t)+k_{2} b(L, t)
$$

at the end $x=L$ and for the normal modes

$$
\mathcal{U}_{i}(0)=0=\mathcal{U}_{i}^{\prime}(0)
$$

at the end $x=0$ and

$$
\mathcal{U}_{i}^{\prime \prime}(L)-k_{1} \mathcal{U}_{i}^{\prime}(L)=0=\mathcal{U}_{i}^{\prime \prime \prime}(L)+k_{2} u_{i}(L)
$$

at end $x=L$ which implies that

$$
\mathcal{U}_{j}(0)=0=\mathcal{U}_{j}^{\prime}(0)
$$

and

$$
u_{j}^{\prime \prime}(L)-k_{1} u_{j}^{\prime}(L)=0=\mathcal{U}_{j}^{\prime \prime \prime}(L)+k_{2} u_{j}(L)
$$

Using equations (59) and (60), it can be shown that at $x=0$,

$$
A_{i}=-C_{i} \text { and } B_{i}=-1
$$

and at $x=L$, using (64)

$A_{i}=\frac{\frac{\phi_{i}}{L}\left[\sin \phi_{i}+\sinh \phi_{i}\right]+k_{1}\left[\cos \phi_{i}-\cosh \phi_{i}\right]}{\frac{\phi_{i}}{L}\left[\cos \phi_{i}+\cosh \phi_{i}\right]-k_{1}\left[\sin \phi_{i}+\sinh \phi_{i}\right]}=\frac{\frac{\phi_{i}^{3}}{L^{3}}\left[\cos \phi_{i}+\cosh \phi_{i}\right]+k_{2}\left[\sinh \phi_{i}-\sin \phi_{i}\right]}{\frac{\phi_{i}^{3}}{L^{3}}\left[\sin \phi_{i}-\sinh \phi_{i}\right]+k_{2}\left[\cos \phi_{i}-\cosh \phi_{i}\right]}$ $=-C_{i}$

From (66) one obtains $\tan \phi_{i}=\tanh \phi_{i}$

Hence, we have $\phi_{1}=3.927, \phi_{2}=7.069, \phi_{3}=10.21 \ldots$

Putting equations (65), (66) and (68) into equations (41)and (53), one obtains the displacement response respectively to a moving force and a moving mass of clamped-elastic ends Rayleigh beam on a variable foundation.

\section{Elastically Supported Conditions at Both Ends}

For the case when the beam is elastically supported both at $x=0$ and $x=L$, the conditions are expressed as

$$
b^{\prime \prime}(0, t)-k_{1} b^{\prime}(0, t)=0=b^{\prime \prime \prime}(0, t)+k_{2} b(0, t)
$$

at $x=0$ and

$$
b^{\prime \prime}(L, t)-k_{1} b^{\prime}(L, t)=0=b^{\prime \prime \prime}(L, t)+k_{2} b(L, t)
$$

at $x=L$

Similarly, for normal modes

$$
u_{i}^{\prime \prime}(0)-k_{1} u_{i}^{\prime}(0)=0=\mathcal{U}_{i}^{\prime \prime \prime}(0)+k_{2} u_{i}(0)
$$

at $x=0$ and

$$
\mathcal{U}_{i}^{\prime \prime}(L)-k_{1} \mathcal{U}_{i}^{\prime}(L)=0=\mathcal{U}_{i}^{\prime \prime \prime}(L)+k_{2} \mathcal{U}_{i}(L)
$$

at $x=L$ which implies that

Citation: Adeoye AS and Awodola TO. Dynamic response to moving distributed masses of pre-stressed uniform rayleigh beam resting on variable elastic pasternak foundation (2018) Edelweiss Appli Sci Tech 2: $1-9$ 


$$
u_{j}^{\prime \prime}(0)-k_{1} u_{j}^{\prime}(0)=0=\mathcal{U}_{j}^{\prime \prime \prime}(0)+k_{2} u_{j}(0)
$$

at $x=0$ and

$$
u_{j}^{\prime \prime}(L)-k_{1} u_{j}^{\prime}(L)=0=\mathcal{U}_{j}^{\prime \prime \prime}(L)+k_{2} u_{j}(L)
$$

at $x=L$ using (71) and (72), it can be shown that

$$
\begin{gathered}
C_{I}=\frac{\left[\frac{\phi_{i}}{L}-k_{1} r_{2}\right] \sin \phi_{i}+\left[k_{1}+\frac{r_{2} \phi_{i}}{L}\right] \cos \phi_{i}-\frac{r_{1} \phi_{i}}{L} \sinh \phi_{i}+k_{1} r_{1} \cosh \phi_{i}}{k_{1} r_{1} \sin \phi_{i}-\frac{r_{1} \phi_{i}}{L} \cos \phi_{i}+\left[\frac{r_{3} \phi_{i}}{L}-k_{1}\right] \sinh \phi_{i}+\left[\frac{\phi_{i}}{L}-k_{1} r_{3}\right] \cosh \phi_{i}} \\
=\frac{-\left[\frac{r_{2} \phi_{i}^{3}}{L^{3}}+k_{2}\right] \sin \phi_{i}+\left[\frac{\phi_{i}^{3}}{L^{3}}-k_{2} r_{2}\right] \cos \phi_{i}-k_{2} r_{1} \sinh \phi_{i}-\frac{r_{1} \phi_{i}^{3}}{L^{3}} \cosh \phi_{i}}{\left.\frac{r_{1} \phi_{i}^{2}}{L^{3}} \sin \phi_{i}+k_{2} r_{1} \cos \phi_{i}+\left[\frac{\phi_{i}^{2}}{L^{3}}+k_{2} r_{2}\right] \sinh \phi_{i}+\frac{r_{3} \phi_{i}^{2}}{L^{3}}+k_{2}\right] \cosh \phi_{i}} \\
A_{i}=r_{1} C_{i}+r_{2} \text { and } B_{i}=r_{3} C_{i}+r_{1}
\end{gathered}
$$

Where

$$
r_{1}=\frac{\frac{\phi_{i}^{4}}{L^{4}}+k_{1} k_{2}}{\frac{\phi_{i}^{4}}{L^{4}}-k_{1} k_{2}} ; r_{2}=\frac{-\frac{2 k_{1} \phi_{i}^{3}}{L^{3}}}{\frac{\phi_{i}^{4}}{L^{4}}-k_{1} k_{2}} \text { and } r_{3}=\frac{-\frac{2 k_{1} \phi_{i}}{L}}{\frac{\phi_{i}^{4}}{L^{4}}-k_{1} k_{2}}
$$

Using equations (75), (76) and (77), the frequency equation for the dynamical problem is obtained as

$$
\tan \phi_{i}=\tanh \phi_{i}
$$

Hence. We have

$$
\phi_{1}=3.927, \phi_{2}=7.069, \phi_{3}=10.21 \ldots
$$

Substituting equations (75), (76), (77) and (78) into equations (41) and (53) one obtains the displacement response respectively to a moving force and a moving mass of Rayleigh beam elastically supported at both ends and resting on a variable foundation.

\section{Numerical Results and Discussions}

To illustrate the analysis presented in this work, the uniform Rayleigh beam is taken to be of length $\mathrm{L}=12.192 \mathrm{~m}$, the load velocity $\mathrm{c}=8.128$ $\mathrm{m} / \mathrm{s}$ and modulus of elasticity $E=2.109 \times 10^{9} \mathrm{~kg} / \mathrm{m}$, the moment of inertia $I_{o}=2.87698 \times 10^{-3} \mathrm{~m}^{4}$.

\section{Graphs for Free-Elastic Boundary Conditions}

Figures 6.1 and 6.2 display the effect of axial force $\mathrm{N}$ on the deflection profile of free elastic Rayleigh beam under the action of load moving at constant velocity in both cases of moving distributed forces and moving distributed masses respectively. The graphs show that the response amplitude decreases as the value of $\mathrm{N}$ increases.

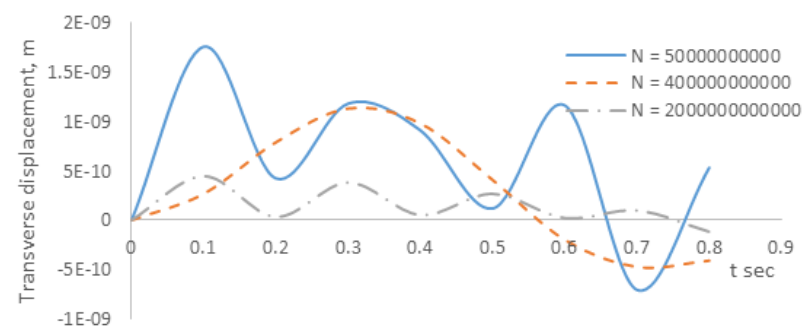

Figure 6.1: Deflection profile of a free elastic uniform Rayleigh beam on variable foundation and traversed by moving distributed force for various values of $\boldsymbol{N}$.

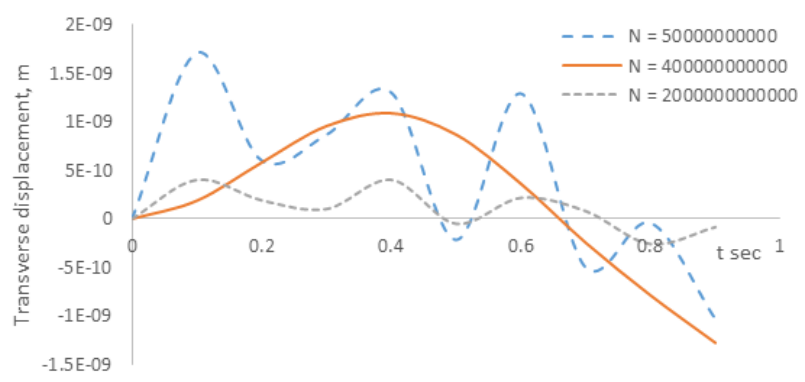

Figure 6.2: Deflection profile of a free elastic uniform Rayleigh beam on variable foundation and traversed by moving distributed mass for various values of $\boldsymbol{N}$.

Figures 6.3 and 6.4 display the effect of rotatory inertia $R$ on the deflection profile of free elastic Rayleigh beam under the action of load moving at constant velocity in both cases of moving distributed forces and moving distributed masses respectively. The graphs show that the response amplitude decreases as the value of $\mathrm{R}$ increases. Figures 6.5 and 6.6 display the effect of foundation modulus So on the deflection profile of free elastic Rayleigh beam under the action of load moving at constant velocity in both cases of moving distributed forces and moving distributed masses respectively. The graphs show that the response amplitude decreases as the value of So increases.

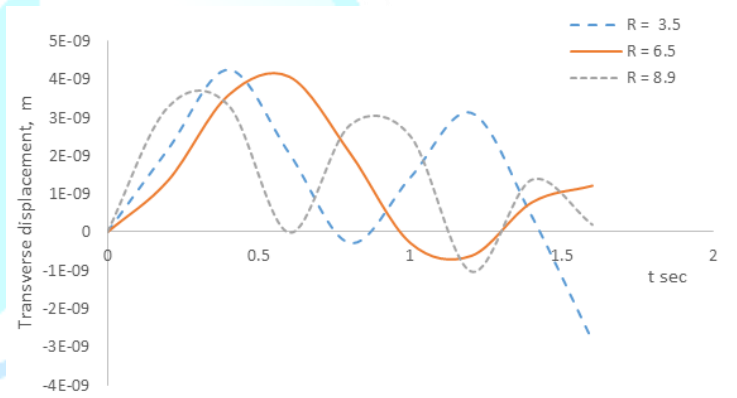

Figure 6.3: Deflection profile of a free elastic uniform Rayleigh beam on variable foundation and traversed by moving distributed force for various values of $\boldsymbol{R}$.

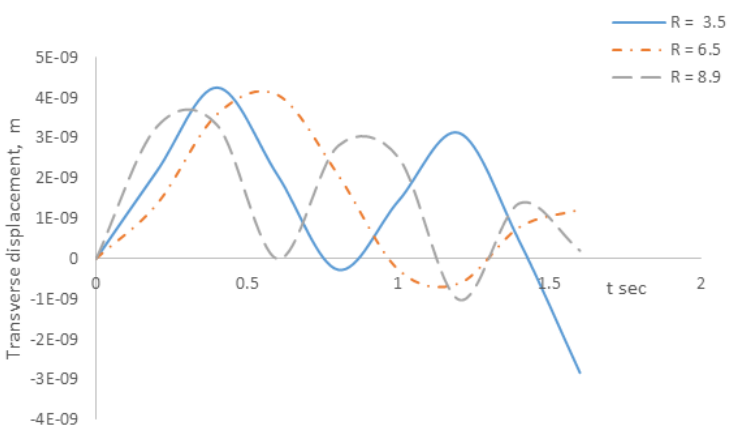

Figure 6.4: Deflection profile of a free elastic uniform Rayleigh beam on variable foundation and traversed by moving distributed force for various values of $\boldsymbol{R}$.

Citation: Adeoye AS and Awodola TO. Dynamic response to moving distributed masses of pre-stressed uniform rayleigh beam resting on variable elastic pasternak foundation (2018) Edelweiss Appli Sci Tech 2: $1-9$ 


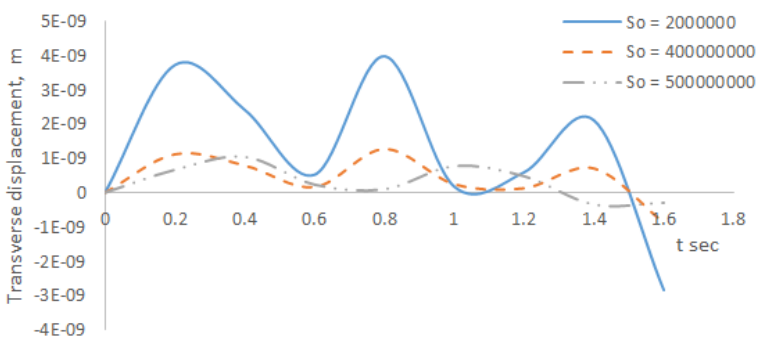

Figure 6.5: Deflection profile of a free elastic uniform Rayleigh beam on variable foundation and traversed by moving distributed forces for various values of $\boldsymbol{S o}$

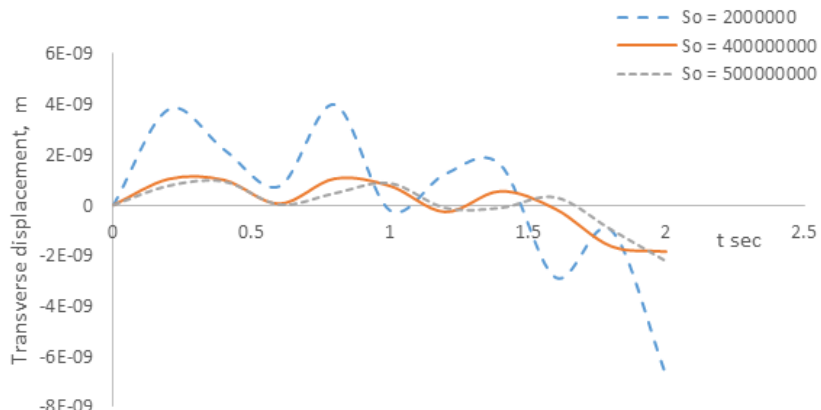

Figure 6.6: Deflection profile of a free elastic uniform Rayleigh beam on variable foundation and traversed by moving distributed mass for various values of $\boldsymbol{S o}$

Figures 6.7 and 6.8 display the effect of shear modulus $\mathrm{H}$ on the deflection profile of free elastic Rayleigh beam under the action of load moving at constant velocity in both cases of moving distributed forces and moving distributed masses respectively. The graphs show that the response amplitude decreases as the value of $\mathrm{H}$ increases.

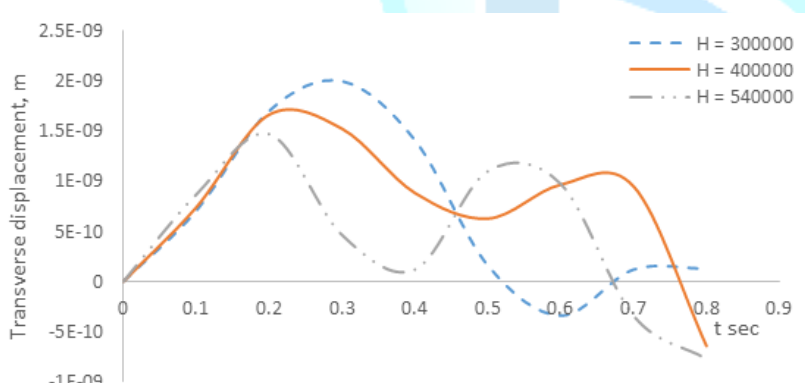

Figure 6.7: Deflection profile of a free elastic uniform Rayleigh beam on variable foundation and traversed by moving distributed force for various values of $\mathrm{H}$.

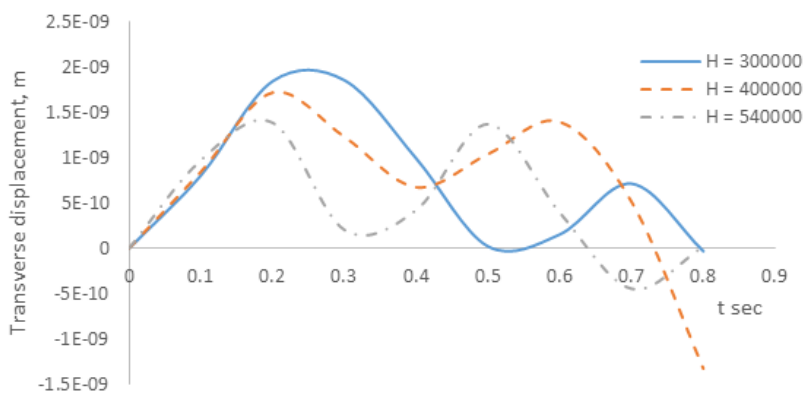

Figure 6.8: Deflection profile of a free elastic uniform Rayleigh beam on variable foundation and traversed by moving distributed mass for various values of $\mathcal{H}$.

Figure 6.9: shows the comparison of the moving distributed forces and moving distributed masses for fixed values of Ho, N, So and R.

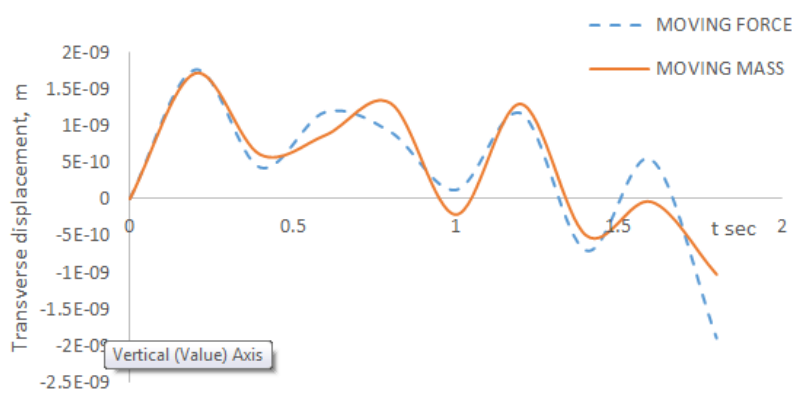

Figure 6.9: Comparison of the deflection profile of moving force and moving mass for a free elastic uniform Rayleigh beam.

\section{Graphs for Elastic-Elastic Boundary Conditions}

Figures 6.10 and 6.11 display the effect of axial force $\mathrm{N}$ on the deflection profile of elastic- elastic Rayleigh beam under the action of load moving at constant velocity in both cases of moving distributed forces and moving distributed masses respectively. The graphs show that the response amplitude decreases as the value of rotatory inertia increases

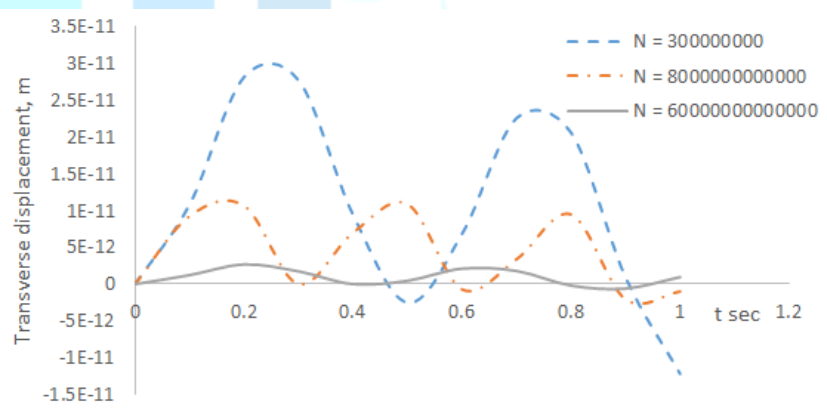

Figure 6.10: Deflection profile of an elastic- elastic uniform Rayleigh beam on variable foundation and traversed by moving distributed forces for various values of $\boldsymbol{N}$.

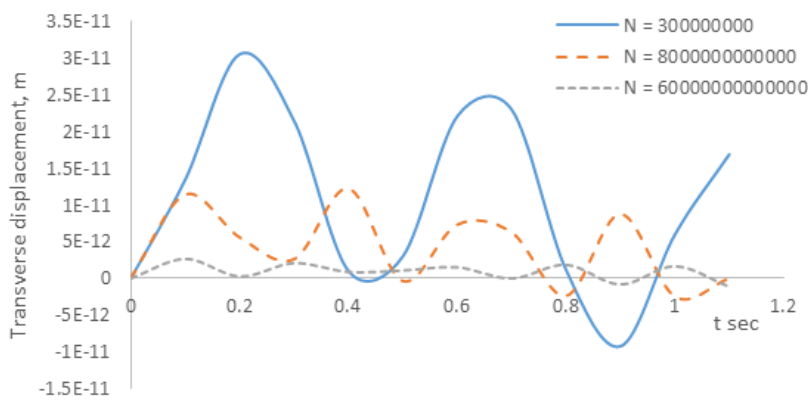

Figure 6.11: Deflection profile of an elastic- elastic uniform Rayleigh beam on variable foundation and traversed by moving distributed forces for various values of $\boldsymbol{N}$ 
Figures 6.12 and 6.13 display the effect of rotatory inertia $R$ on the deflection profile of elastic- elastic Rayleigh beam under the action of load moving at constant velocity in both cases of moving distributed forces and moving distributed masses respectively. The graphs show that the response amplitude decreases as the value of rotatory inertia increases.

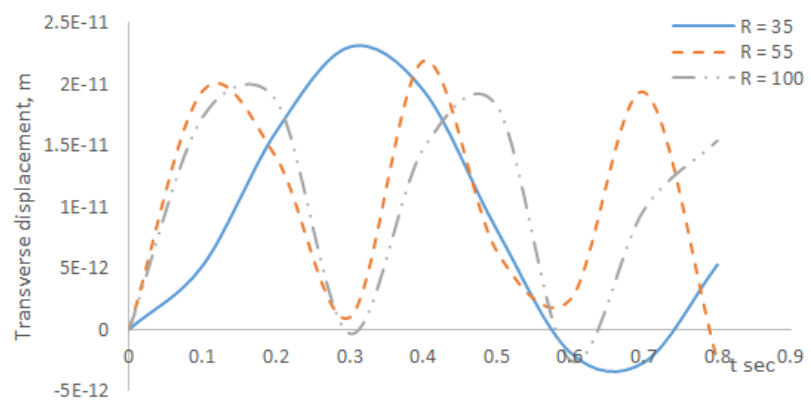

Figure 6.12: Deflection profile of an elastic- elastic uniform Rayleigh beam on variable foundation and traversed by moving distributed forces for various values of $\boldsymbol{R}$

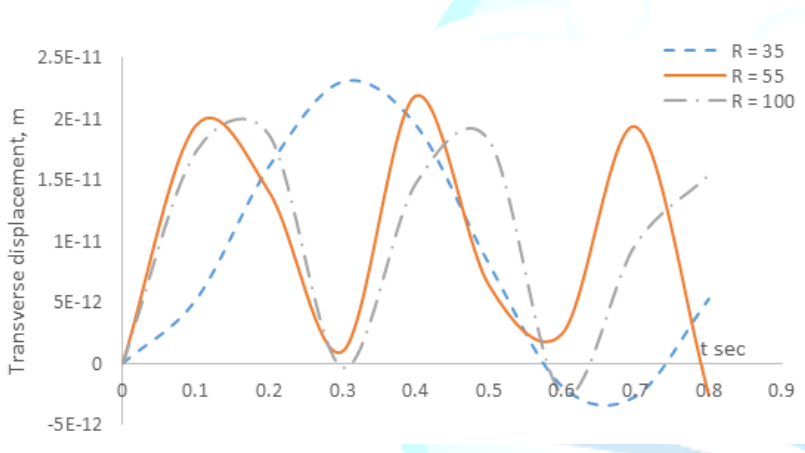

Figure 6.13: Deflection profile of an elastic-elastic uniform Rayleigh beam on variable foundation and traversed by moving distributed mass for various values of $\boldsymbol{R}$

Figures 6.14 and 6.15 display the effect of shear modulus $\mathcal{H}$ on the deflection profile of clamped elastic Rayleigh beam under the action of load moving at constant velocity in both cases of moving distributed forces and moving distributed masses respectively. The graphs show that the response amplitude decreases as the value of $\mathcal{H}$ increases.

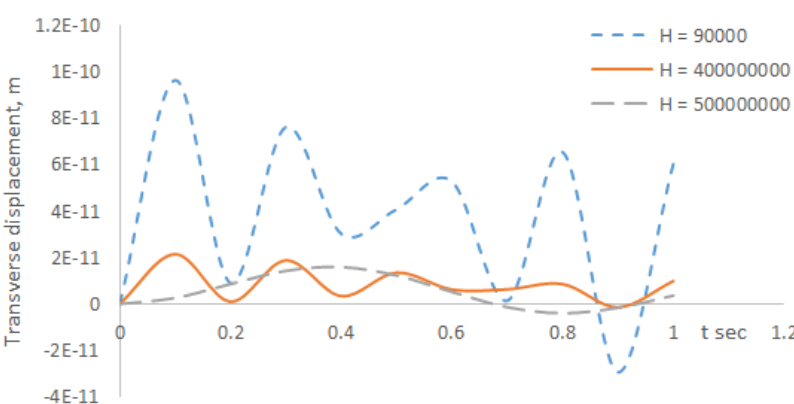

Figure 6.14: Deflection profile of an elastic-elastic uniform Rayleigh beam on variable foundation and traversed by moving distributed force for various values of $\mathcal{H}$.

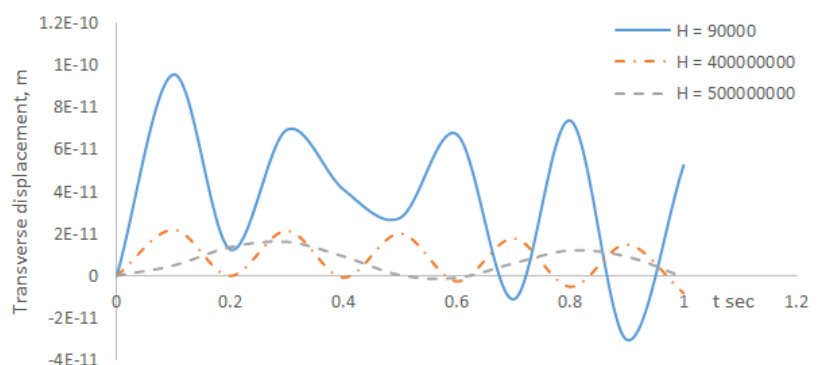

Figure 6.15: Deflection profile of an elastic-elastic uniform Rayleigh beam on variable foundation and traversed by moving distributed masses for various values of $\mathcal{H}$.

Figures 6.16 and 6.17 display the effect of foundation modulus $S o$ on the deflection profile of clamped elastic Rayleigh beam under the action of load moving at constant velocity in both cases of moving distributed forces and moving distributed masses respectively. The graphs show that the response amplitude decreases as the value of $\boldsymbol{S o}$ increases.

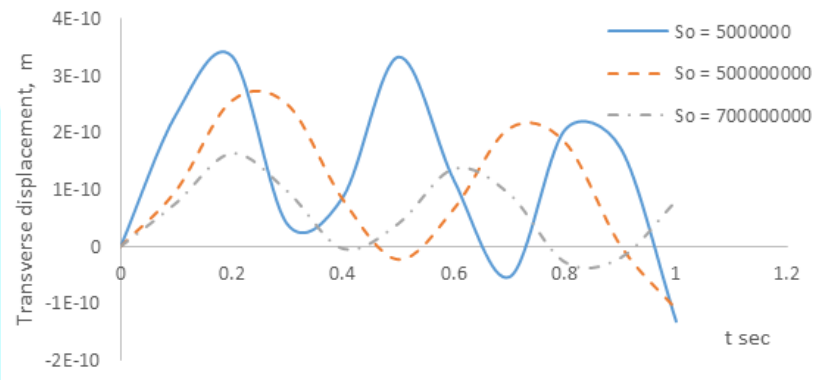

Figure 6.16: Deflection profile of an elastic-elastic uniform Rayleigh beam on variable foundation and traversed by moving distributed force for various values of $\boldsymbol{S o}$.

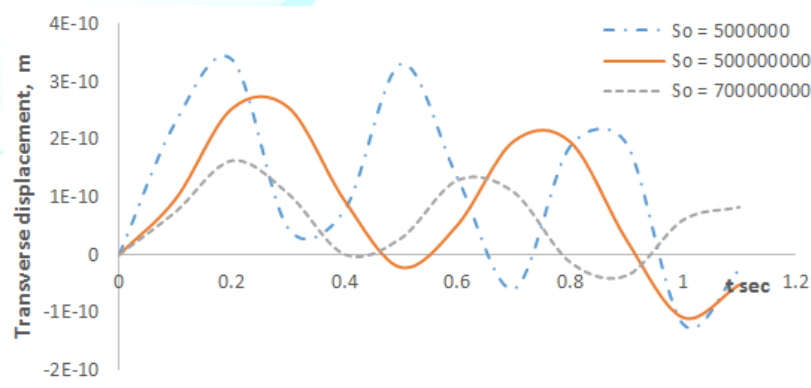

Figure 6.17: Deflection profile of an elastic-elastic uniform Rayleigh beam on variable foundation and traversed by moving distributed mass for various values of $\boldsymbol{S o}$.

Figure 6.18 shows the comparison of the moving distributed forces and moving distributed masses for fixed values of $\boldsymbol{S o}, \boldsymbol{N}, \mathcal{H}$ and $\boldsymbol{R}$.

Citation: Adeoye AS and Awodola TO. Dynamic response to moving distributed masses of pre-stressed uniform rayleigh beam resting on variable elastic pasternak foundation (2018) Edelweiss Appli Sci Tech 2: $1-9$ 


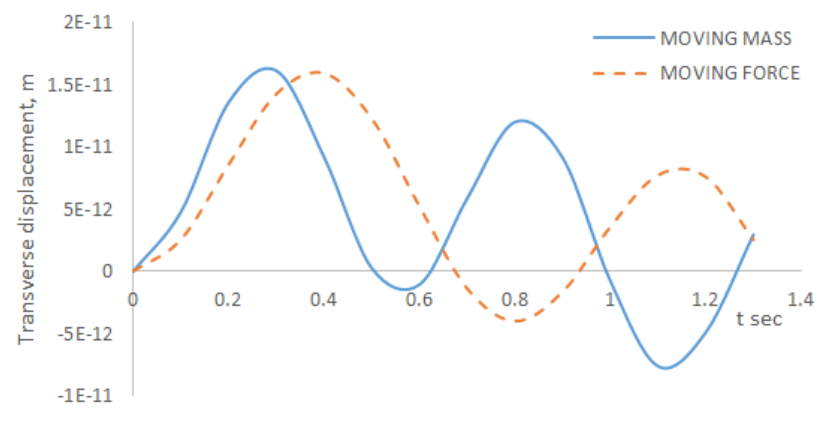

Figure 6.18: Comparison of the deflection profile of moving force and moving mass for elastic-elastic uniform Rayleigh beam for fixed values of So, No, Ro, and $\mathcal{H}$.
9. Oni ST and Omolofe B. Dynamic analysis of a prestressed elastic beam with general boundary conditions under moving loads at varying velocities (2005) J Eng Eng Tech 4: 55-72.

10. Li WL, Zhang X, Du J and Liu Z. An exact series solution for the transverse vibration of rectangular plates with general elastic boundary supports (2009) J Sound Vibrations 321: 171-182. https://doi.org/10.1016/j.jsv.2008.09.035

11. Hsu MH. Vibration analysis of non-uniform beams resting on elastic foundations using the spline collocation method (2009) Tamkang J Sci Eng 12: 113-122.

12. Ismail E. Dynamic response of a beam due to an accelerating moving mass using moving finite element approximation (2011) Math Comput Appli 16: 171-182. https://doi.org/10.3390/mca16010171

13. Kargarmovin $\mathrm{MH}$ and Younesian D. Dynamics of Timoshenko beams on Pasternak foundation under moving load (2004) Mechanics Res Communications 31: 713 -723 . https://doi.org/10.1016/j.mechrescom.2004.05.002

In this research work, the problem associating with the dynamic response to moving distributed masses of pre-stressed uniform Rayleigh beam resting on variable elastic Pasternak foundation has been studied. The closed form solutions of the fourth order partial differential equations with variable and singular co-efficients are obtained for both cases of moving force and moving mass. From the closed form solutions obtained, the conditions of resonance are obtained for both moving force and moving mass. Also from the closed form solutions, the effects of beam parameters such as rotatory inertia, axial force, shear modulus and foundation modulus on the beam for both cases of moving distributed force and moving distributed mass were investigated.

\section{References}

1. Fryba L. Vibration of solids and structures under moving loads (1972) Noordhoff International Publishing Groningen, Groningen, Netherlands.

2. Gbadeyan JA and Dada MS. The dynamic response of plates on pasternak foundation to distributed moving load (2001) J Nigerian Association Math Physics 5: 185-200.

3. Gbadeyan JA and Oni ST. Dynamic behavior of beams and rectangular plates under moving loads (1995) Journal of Solid and Vibration 182: 677-695. https://doi.org/10.1006/jsvi.1995.0226

4. Yavari A, Sarkani S and Reddy JN. Generalized Solution of beams with jump discontinuities on elastic foundations (2001) Archive Appl Mechanics 71: 625-639. https://doi.org/10.1007/s004190100169

5. Yin JH. Closed form solution for reinforced Timoshenko beam on elastic foundation (2000) J Eng Mechanic 126: 868-874. https://doi.org/10.1061/(ASCE)07339399(2000)126:8(868)

6. Teodoru IB. Analysis of beams on elastic foundation: The finite difference approach (2007) 9th Technical Conference for Doctoral Study, Brno University of Technology, Czech Republic.

7. Oni ST and Awodola TO. Vibrations under a moving load of a non-uniform rayleigh beam on variable elastic foundation (2003) J Nigeria Association math Physics 7: 191-206.

8. Awodola TO and Oni ST. Dynamic response to moving masses of rectangular plates with general boundary conditions and resting on variable winkler foundation (2013) Latin Ame J Solids Structures 10: 301-322. http://dx.doi.org/10.1590/S1679-78252013000200005 